\title{
L'insulte comme argument et outil de cadrage dans le mouvement « anti-Sarko »
}

Insult as Argument and Framing Device in the "Anti-Sarko" Movement

\section{Eithan Orkibi}

\section{(2) OpenEdition \\ 1 Journals}

\section{Édition électronique}

URL : http://journals.openedition.org/aad/1335

DOI : $10.4000 /$ aad. 1335

ISSN : 1565-8961

Éditeur

Université de Tel-Aviv

Référence électronique

Eithan Orkibi, «L'insulte comme argument et outil de cadrage dans le mouvement « anti-Sarko » », Argumentation et Analyse du Discours [En ligne], 8 | 2012, mis en ligne le 15 avril 2012, consulté le 23 septembre 2019. URL : http://journals.openedition.org/aad/1335; DOI : 10.4000/aad.1335

Ce document a été généré automatiquement le 23 septembre 2019.

\section{cc)}

Argumentation \& analyse du discours est mis à disposition selon les termes de la licence Creative Commons Attribution - Pas d'Utilisation Commerciale - Pas de Modification 4.0 International. 


\title{
L'insulte comme argument et outil de cadrage dans le mouvement «anti-Sarko»
}

\author{
Insult as Argument and Framing Device in the "Anti-Sarko" Movement
}

\author{
Eithan Orkibi
}

\section{Introduction}

1 "Le nom de Sarkozy provoque un pénible désir d'injure », constate le juriste Stéphane Rials dans une interview consacrée à la multiplication des offenses envers le Président de la République. "L'on croise souvent désormais des gens raisonnables qui "disjonctent", comme on dit, dès que le nom de Sarkozy est prononcé » (Libération, 4 juillet 2009). Ce "désir d'injure» se manifeste également dans l'inflation de faits enregistrés comme délit d'outrage, qui, selon une pétition ${ }^{1}$ diffusée en France en fin 2009, " s'inscrit clairement dans le contexte actuel du "tout-répressif" 》 (Libération, 30 décembre 2008). En 2011, il y a ceux qui croient que Nicolas Sarkozy est l'un des hommes politiques les plus « injuriés » sur la place publique, et peut-être l'un des chefs d'Etat les plus «outragés» des récentes générations politiques en France (Maffesoli 2011 ; Bercoff 2011) $)^{2}$.

2 Comme le montrent Amossy et Koren (2010), l'argumentation politique des adversaires du candidat UMP a été marquée durant la campagne présidentielle de 2007 par une pratique de "diabolisation ». Cette « arme de combat » a consisté à représenter Nicolas Sarkozy comme porteur d'une "idéologie menaçante pour l'identité française " et comme "l'incarnation de tous les maux» (2010: 230). Cette tendance a atteint de nouveaux sommets avec l'élection de Sarkozy. La diabolisation du candidat - et par la suite du Président - à travers l'insulte et l'invective ${ }^{3}$ est devenue une pratique discursive largement partagée dans le discours du nouveau mouvement « anti-Sarko ». 
3 Centré sur l'opposition à Sarkozy, ce mouvement réunit des associations et des militants de diverses tendances politiques, appartenant pour la plupart à la gauche française, mais se déclarant «non partisans ». Comme le formule l'un des acteurs : « il ne s'agit pas ici d'un débat partisan droite/gauche car le danger que représente Nicolas Sarkozy dépasse largement les clivages politiques traditionnels $»^{4}$. Bien que peu institutionnalisé sur le plan organisationnel, ce mouvement se dote néanmoins des ses propres espaces discursifs à travers un certain nombre de portails, sites et blogs sur internet (sarkozyland.org, antisarko.org, sarkostique.blog.net, no.sarko.free.fr, etc.), voire d'un journal imprimé : Le Sarkophage (29 numéros sont parus depuis 2007).

4 Une partie de la production discursive issue du mouvement a pour objectif «la reconstruction de la gauche $»^{5}$ et se donne pour mission $d^{\prime}$ ' inventer un langage politique capable de concilier les contraintes environnementales et notre souci de justice sociale $»^{6}$. De ce fait, elle dénonce certaines expressions, comme le slogan "sarko-facho », jugé "totalement infécond car politiquement faux » ou "tout sauf Sarkozy ", conçu comme "erroné car il exonère les partis de gauche de leurs responsabilités dans notre défaite ${ }^{7}$. Néanmoins, une autre partie de cette production discursive poursuit le travail de discrédit de Sarkozy à travers la création systématique et intensive de slogans, illustrations, graffitis et affiches où le Président est la cible d'attaques personnelles particulièrement virulentes. La «blogosphère ", arène de prédilection pour la création et la diffusion des ressources « anti-Sarko », est riche en initiatives invitant les utilisateurs à envoyer et partager leurs œuvres ${ }^{8}$. D'autres sites et blogs se spécialisent dans la création et la mise à jour de clips, affiches, caricatures et slogans ciblant Nicolas Sarkozy ${ }^{9}$.

5 Si le discrédit jeté sur le Président par le mouvement anti-Sarko s'effectue ainsi à travers plusieurs dispositifs et nouvelles méthodes d'expression, il se développe également sur le plan thématique. A la diabolisation destinée à représenter Sarkozy comme un danger pour la démocratie s'ajoute la dévalorisation qu'entraînent la ridiculisation du personnage du Président et une moquerie cinglante et souvent obscène. Dans ce cadre, le milieu virtuel du mouvement anti-Sarko est largement associé à la floraison du genre satirique connu sous le nom de «fake » (faux), à savoir le détournement pastiche des journaux, des images ou des affiches. Le mouvement antiSarko devient ainsi une véritable "fabrique d'insultes » dont la cible principale est Nicolas Sarkozy en tant qu'homme politique, et en tant qu'homme tout court.

6 Cet article se propose d'exploiter les notions et les catégories élaborées par la rhétorique des mouvements sociaux pour étudier les fonctions rhétoriques de l'insulte dans le discours du mouvement anti-Sarko, et plus particulièrement dans les manifestations qui associent étroitement le visuel au verbal. Il offre une catégorisation de ses diverses modalités, tout en éclairant la façon dont elles s'insèrent dans une véritable structure argumentative - soulignant ainsi le lien souvent méconnu entre insulte et argumentation. Qui plus est, il postule que l'usage de l'insulte, en tant que pratique discursive, permet à un mouvement composé de plusieurs courants idéologiques et politiques - parfois contradictoires - de construire un ensemble de significations partagées. L'analyse montrera que l'insulte constitue et reflète le consensus interne du mouvement en offrant un outil de cadrage à l'aide duquel les différentes fractions définissent ensemble la cause poursuivie par le mouvement. Une présentation théorique précédera l'analyse stratégique et thématique d'un corpus composé de divers exemples. 


\section{L'insulte dans la rhétorique des mouvements sociaux}

7 Englobant différentes formes d'agression verbale et non-verbale, l'insulte est une pratique discursive qui offre une représentation symbolique de la confrontation entre un mouvement social et ses différents adversaires, notamment le Pouvoir (Simons 1972). Etant une figure de violence verbale, elle constitue un bon séismographe de la montée en tension dans un contexte d'agitation et de mobilisation (Fracchiolla \& Moïse 2009), mais elle marque aussi souvent la production discursive d'un mouvement à travers toutes les étapes de son évolution, de son émergence à sa dispersion.

Avant de revenir aux fonctions de l'insulte dans la rhétorique des mouvements sociaux $^{10}$ (désormais RMS), certaines précisions s'imposent. La première est qu'à l'instar de toute autre parole provenant du mouvement, l'insulte est conçue comme l'expression d'un collectif. Même lorsqu'elle est prononcée par un individu - un manifestant portant une affiche ou prononçant un discours - l'insulte représente l'ensemble des individus dont est composé le mouvement, qui est, par définition, un locuteur collectif.

9 Puisqu'elle représente la manière dont le mouvement conçoit la cible (individu ou groupe), l'insulte est par définition plus essentialiste que situationnelle. Selon Ernotte et Rosier (2004: 37), l'insulte essentialiste est celle qui, « hors de toute motivation par le contexte, met nominalement en cause l'individu interpellé dans son essence ». Cette "essence» est très souvent considérée par le mouvement comme un élément constitutif de la réalité problématique mise en cause.

10 Dans ce contexte, l'insulte peut être de type dialogal (l'insulté est présent) ou délocutive (l'insulté est absent), pour reprendre la typologie d'Ernotte et Rosier (ibid.). Elle est dialogale, par exemple, lorsqu'un représentant du Pouvoir est victime d'un attentat-pâtissier (« entartage », ou pieing en anglais) ou lorsque les manifestants crient «Fachos! Cochons !» à l'encontre des policiers. Or, sauf dans le cas particulier d'une confrontation directe entre les militants et la cible (la police n'étant souvent qu'une instance représentative de la cible véritable, le plus souvent le Pouvoir), le mouvement social manie plutôt des insultes délocutives (ou «indirectes » dans la terminologie de Fracchiolla et Moïse, 2009). Etant une pratique de subversion, l'essentiel ici est l'effet de l'insulte sur le public, par exemple un électorat, comme l'a constaté Herbert Marcuse dans son Essay on Liberation:

If, for example, the highest executives of the nation or the state are called, not President X or Governor Y but pig X and pig Y, and if what they say in campaign speeches is rendered as "oink, oink," this offensive designation is used to deprive them of the aura of public servants or leaders who have only the common interest in mind (1969: 36).

11 Dans la mesure où sa bonne réputation est la source de la légitimité, de l'autorité et du prestige de l'homme politique, l'insulte n'est efficace que si le public (le tiers) atteste de la manière dont elle a affecté le renom de la cible. Cette configuration contribue à la création d'un "effet Cyrano", à savoir, selon Rosier (2006), l'inflation et la théâtralisation de l'insulte par la présence du «tiers écoutant» (76). Fracchiolla et Moïse (2009) constatent en plus que 
L'injure indirecte est d'autant plus forte ici qu'elle est cautionnée par l'injuriaire (celui qui est témoin de l'insulte), les médias, le peuple, la France entière. L'insulte fonctionne alors avec le consentement de l'injuriaire séduit par l'injurieur (les manifestants), mais dans une forte agressivité, l'injurié (les hommes politiques) étant absents et délocutés dans une forme d'adresse par le nom propre (2009: 118).

Ainsi, notre point de départ pose que dans la RMS, l'insultant est collectif, l'insulte est essentialiste, et son efficacité repose sur la présence d'un tiers écoutant. C'est en vertu de ces traits caractéristiques que l'insulte est considérée comme une pratique discursive liée aux procédés de construction identitaire et de renforcement de la solidarité au sein du mouvement. En premier lieu, elle est associée à la stratégie de polarisation, ainsi définie dans l'ouvrage classique de Bowers et al. :

Polarization assumes that any individual who has not committed to the agitation supports the establishment. [...] The strategy of polarization encompasses tactics designed to move the individual into the ranks - to force a conscious choice between agitation and control. [...] At the polarization stage, agitators are no longer interested in addressing nuances. [...] Painting issues as black and white - for and against - defines this stage (1993 [1971]): 34).

La polarisation est une stratégie rhétorique dont l'objectif principal est d'établir une distinction nette et catégorique entre «nous » (le mouvement) et « eux », à savoir ceux qui ne sont pas avec "nous ", quelle que soit leur affiliation : le public non engagé, les cadres non mobilisés, les militants d'un contre mouvement, les représentants du Pouvoir. Dans les termes de Stewart et al. (2007 [1984] : 164), il s'agit d'une stratégie qui "divise afin de réunir ». La polarisation peut se manifester à travers un discours qui dévalorise une collectivité toute entière ou, comme le suggèrent King et Anderson, par " a careful selection of those images that will undermine the ethos of competing groups, ideologies, or institutions » (1971:244, également cité par Stewart et al. : ibid.). Dans ce sens, le discours de polarisation cherche à dévaloriser tous ceux qui ne font pas (encore) partie du mouvement (« vous êtes avec nous ou contre nous!») et impose par là même un choix éthique au destinataire.

Or, la polarisation peut également se manifester à travers la désignation d'une cible plus précise, que ce soit un problème particulier ou, plus souvent, un individu spécifique (ce que Bowers et al. dénomment flag issues ou flag individual). La contestation du mouvement est orientée vers une cible qui est alors susceptible, d'un coté d'attirer l'attention du public, et de l'autre de canaliser l'énergie des militants. Le mouvement se rassemble ainsi autour d'un ennemi commun soigneusement sélectionné : «the person chosen must be viewed by members of the dissenting group as being worthy of attack and must be vulnerable to attack» (ibid. : 35). L'attaque s'achève souvent par l'invention d'un "jargon dérogatoire», qui devient une partie intégrante du "vocabulaire spécialisé » du mouvement, et un élément constituant de sa cohésion interne (ibid. : 36).

Or, en plus de réunir les militants autour d'un ennemi ou de canaliser leur frustration en direction d'un bouc émissaire, la polarisation permet de créer une "réalité symbolique »:

Social movements employ verbal symbols to define good and bad and to identify with a set of social values or specific audience. Labeling is an act of judgment; it makes positive or negative associations. We act towards people, groups, and objects based on our understanding of them, and labels tell us what is important and what to expect (Stewart et al. 2007 [1984] : 170). 
Ainsi, la diabolisation (vilification) et la ridiculisation sont toutes deux associées à la pratique d'étiquetage (labeling) et à l'insulte (name-calling), et conçues par Stewart et al. (ibid.: 170-176) comme des tactiques langagières destinées à construire un sens commun et à fournir une série de significations partagées propre à l'action protestataire. Vanderford (1989) a constaté que la diabolisation permet de désigner une force adversaire spécifique qui, d'un côté, est dotée d'une puissance tellement diabolique qu'elle en devient alarmante, mais qui en même temps désigne une cible claire et précise pour l'action. Les membres du mouvement peuvent sentir alors que leur cible est plus concrète et leur lutte plus réalisable. En plus, la diabolisation d'un ennemi qui incarne tous les maux permet aux militants de se définir comme ceux qui luttent pour la justice contre Satan. Dans ce sens, à travers l'insulte, le mouvement se donne à voir comme une force dotée d'une mission: il se doit de réparer les torts perpétrés au détriment de la société (1989 : 166-167).

17 A l'instar de la diabolisation, la ridiculisation permet aux membres du mouvement de s'approprier toutes les qualités contraires à celles de la cible : c'est en la définissant comme illogique ou inhumaine qu'ils se définissent eux-mêmes comme logiques ou humains. La pratique de la ridiculisation procure ainsi un espace discursif où celui qui tourne l'autre en dérision se présente comme supérieur à lui sur le plan épistémologique et moral. C'est dans ces espaces que la hiérarchie du monde "réel ", où la cible occupe une position d'autorité et de pouvoir, est renversée au profit des « simples citoyens ».

18 La ridiculisation constitue un «outil rhétorique " particulièrement efficace puisque n'étant pas un argument à proprement parler - elle est relativement difficile à réfuter. En fait, sans forcément attribuer des mauvaises intentions à la cible, la ridiculisation

attacks the basic worth and credibility of persons and ideas and thus challenges assigned or claimed legitimacy. Social movements employ ridicule to make fun of a person, group, place, thing, action or idea for being inconsistent, illogical, inept, silly, monstrous or inhuman (Stewart et al., 2007 [1984] :172).

L'usage d'une langue obscène, caractéristique de la rhétorique de polarisation, est tout particulièrement associé à la reprise du contrôle de l'espace public par «le peuple ». Il renvoie, comme le constatent Fracchiolla et Moïse (2009:119), aux effets de carnaval qui « jouent sur le renversement des rapports de pouvoir et des idéologies ». La parole obscène, "forme ultime du conflit symbolique » (Stewart et al., 2007 [1984] : 174), est également la forme ultime de ce que Rosier (2006: 76) dénomme l'inflation et la théâtralisation de l'insulte. Ames (1990) pose que
uttering an obscenity is an illocutionary act that violates a taboo of the official culture and in doing so silently acknowledges allegiance with the entire counterculture of the dispossessed, those for whom the words are not threatening but comforting. Since so much of the power of the Elite arises from their control of authoritative discourse, the breaking of discursive decorum possesses at least symbolic significance. It participates in the comic inversion of propriety and hierarchy associated with the carnival or festive element of nonofficial culture (196).

Dans un essai fondateur sur la diatribe dans la RMS, Windt (1972) postule que l'obscénité est un choix rhétorique qui symbolise le fait que, pour les manifestants, le temps du "débat » est révolu. L'usage du mot " obscène » marque une rupture avec le decorum discursif imposé par l'élite, et le rejet de son mode de raisonnement. En effet, c'est la communication argumentative tout entière qui est contestée, puisqu'elle est 
perçue comme mise à mal par ceux qui dominent l'espace discursif. Windt se réfère à Noam Chomsky qui écrit :

By entering the arena of argument and counter-argument, of technical feasibility and tactics, of footnotes and citations, by accepting the presumption of legitimacy of debate on certain issues, one has already lost one's humanity. [...] There may have been a time when American policy in Vietnam was a debatable matter. This time is long past. [...] The war is simply an obscenity, a depraved act by weak and miserable men (1969: 9).

21 Ainsi, l'obscénité transforme la manifestation en diatribe, forme de satire subversive développée par les cyniques qui, au deuxième siècle av. J.-C., ont parcouru la Grèce pour prêcher leur critique morale de la société. La diatribe est caractérisée par l'usage d'anecdotes et de sketchs, par la parodie et l'obscénité, et son ambition est de critiquer, d'amuser et de choquer (Kennedy 1999). Tandis que l'orateur classique doit projeter à travers son discours un ethos conforme aux attentes de l'auditoire, en respectant les valeurs et les normes dominantes, celui qui prononce une diatribe rejette ces principes dans la mesure où ils représentent la manière dont les normes communicationnelles sont subordonnées à l'immoralité de la société. Il cherche en fait à démasquer la corruption de la société par un discours qui souhaite

to assault sensibilities, to turn thought upside-down, to turn social mores insideout, to commit in language the very same barbarisms one condemns in society. [...] The diatribe is to conventional speeches what Alice's adventures in Wonderland are to conventional life. Logic is inverted; assumptions are reversed; the unexpected is not unusual. [...] The diatribe is to rhetoric what satire is to literature (Windt 1972: 7-8).

C'est donc à travers l'obscénité que le manifestant tente de refléter l'immoralité de la société, et ceci dans l'hypothèse que les normes discursives conventionnelles sont désormais inutiles puisque subordonnées à cette même société corrompue. On se situe donc sur le plan «spectaculaire » du mouvement social : à travers un style marqué par l'obscénité, il offre aux spectateurs, les by-standers, un spectacle où le dégoût face à l'obscénité du manifestant est, en dernière instance, un dégoût face à l'obscénité de la société. Et c'est cette critique morale qui permet l'extension du domaine de la contestation: elle ne s'en prend plus à telle ou telle politique, mais aux principes organisationnels les plus fondamentaux de la société; il ne s'agit plus d'un simple désaccord ou "lutte des opinions", mais d'une rupture profonde entre les militants, d'un côté, et le système de valeurs représenté par l'ennemi du mouvement, de l'autre.

\section{Corpus}

On se penchera ici sur les produits discursifs dominés par une «visée insultante » explicitement marquée. A l'inverse des textes programmatiques (comme les pétitions ou les articles d'opinion), qui peuvent comprendre une "dimension insultante ", notre analyse réunit des textes où l'insulte prime et constitue «la raison d'être » du produit discursif. Sont analysés trois genres dominants: autocollants (stickers), figurant une icône et parfois un très court slogan; affiches, présentant souvent une image et un texte plus élaboré (slogan long, phrase, etc.); et «humour anti-Sarko ", à savoir des pastiches et détournements.

Les matériaux issus du courant « humour anti-Sarko » consistent, dans la plupart des cas, en illustrations qui s'apparentent dans leur forme au genre de la caricature. 
Comme elle, les illustrations anti-Sarko expriment, à travers le rire, une critique politique dans un style excessif et parodique. Or, malgré cette proximité entre la caricature politique et l'humour anti-Sarko, il y a lieu d'établir deux distinctions importantes. D'abord, la caricature politique, genre culturellement institutionnalisé, est publiée régulièrement dans la presse, et exprime une critique politique envers les institutions et le Pouvoir, quelle que soit sa tendance politique. Si l'humour anti-Sarko reprend le style caricatural, il l'utilise cependant dans le cadre d'un mouvement social dont l'ambition n'est pas de critiquer le comportement du Pouvoir mais d'éliminer un homme politique particulier. L'humour anti-Sarko est issu de sites internet, blogs et associations entièrement consacrés à cette seule tâche. Deuxièmement, la caricature, publiée régulièrement dans les quotidiens ou les hebdomadaires, exprime une critique par rapport aux événements courants: elle réagit au contexte immédiat. L'humour anti-Sarko, en revanche, exprime une critique plutôt « essentialiste ", plus globale dans sa nature. Son efficacité et son effet humoristiques sont désormais indépendants de l'actualité courante.

Les produits discursifs émanent des acteurs « officiels » du mouvement "anti-Sarko » (association plus ou moins organisée), ainsi que des acteurs indépendants créant et diffusant des matériaux de propagande à titre plutôt individuel (blogs personnels, etc.). Nous nous concentrons sur des matériaux accessibles sur internet, préparés parfois à l'occasion d'une mobilisation ponctuelle, mais constamment affichés et disponibles : ils font partie du travail constant de la «mobilisation de consensus » du mouvement. Les exemples ont été tirés des deux « réservoirs " qui hébergent et diffusent des dizaines des produits chacun (dont un très grand nombre circule désormais dans d'autres sites) :

1. le site du Comité de Résistance Citoyenne (CRC), «CRC Le blog »"11 qui met à la disposition des utilisateurs plusieurs matériaux regroupés sous la rubrique «Album photos» et sur sa page respective sur Facebook (" Affiches anti-Sarko »);

2. le «Sarko-Show »12, blog d'humour d'un artiste créant des pastiches depuis 2006. Le blog comprend des archives contenant des centaines de dessins classés selon un critère chronologique ;

3. à part ces deux sites centraux, nous avons consulté et utilisé les matériaux disponibles sur le site «Anti-Sarko »", animé par l'association Ré-So (réformistes et solidaires), ainsi que les blogs « SarkoFrance », « SarkowyLand » « Sarkostique » et « Sarkozix » ${ }^{14}$.

\section{Cadre méthodologique}

Notre analyse aborde une optique qui s'intéresse non seulement à l'usage stratégique de l'insulte comme "arme de combat ", mais aussi à ses fonctions comme instrument de construction du sens dans la RMS. Nous nous inspirons, au niveau du traitement des données, de la théorie de l'analyse de cadres (frame perspective) qui a cours en sociologie des mouvements sociaux. Cette théorie, développée dans l'objectif de faciliter l'étude des « raisons de militer et de résister [et] des motifs de critiquer et de contester », se focalise sur des « situations d'épreuve, où se confrontent des discours de dénonciation d'injustice et de revendication de droits, où sont énoncés des principes éthiques ou civiques, esthétiques ou juridiques » (Cefaï \& Trom, 2001 : 11-12). Il est postulé ainsi que les mouvements sociaux sont producteurs et diffuseurs de signification, et qu'ils s'engagent dans un « travail de signification » où le sens est attribué, la signification est construite, et les interprétations sont développées afin d'aboutir à l'adhésion et à la 
mobilisation des auditoires visés (Snow 2001: 28). Ce processus est dénommé " cadrages » (framing) car il produit les " cadres de l'action collective ", ainsi définis par Snow et Benford :

An interpretive schema that simplifies and condenses the world out there by selectively punctuating and encoding objects, situations, events, experiences, and sequences of actions within one's present or past environments (1992:137).

La théorie des cadres pose qu'en l'absence d'un cadre efficace, aucune mobilisation n'est possible. Pour que le mouvement puisse réaliser ses objectifs - recruter des membres, collaborer avec d'autres mouvements, mobiliser les militants en vue d'une action - il doit " encadrer » sa cause, son idéologie et son action de manière à gagner l'adhésion des différents destinataires (Benford \& Snow 2000). Or, par «cadres de l'action ", on ne se réfère pas exclusivement à ceux qui sont stratégiquement construits en vue d'une mobilisation immédiate pour une action précise. Klandermans suggère que la mobilisation à l'action est précédée par une "mobilisation de consensus " (consensus mobilization), à savoir, « a process through which a social movement tries to obtain support for its viewpoint » (1984: 586). Il s'agit ainsi d'un travail de longue durée, durant lequel le mouvement propage sa critique sur une réalité problématique, tout en indiquant qu'une action collective peut aboutir à un changement social (Klandermans \& Oegema, 1987 : 519).

Snow et Benford (1988) établissent une distinction entre trois types de cadres : (a) cadre diagnostique, où le " problème ", sa cause et son ampleur sont définis et caractérisés, et la responsabilité et la culpabilité attribuées ; (b) cadre pronostique, où les objectifs sont formulés, des solutions proposées et des méthodes d'action prescrites; (c) cadre "motivationnel», le cadre qui définit la motivation à participer et renforce l'identification à la cause du mouvement. Best $(1990,2008)$ reprend ce triple modèle et l'intègre au modèle d'argumentation de Toulmin (voir de Jong 2008). Il crée ainsi un modèle pour la construction des cadres des problèmes publics : le cadre diagnostique constitue les "données» (ground ou data), le cadre pronostique constitue la « conclusion» (conclusion ou claim) et le cadre motivationnel constitue « la garantie » (warrant) :

\section{Données \\ Informations et preuves sur la situation perturbante - exemples typiques, statistiques, etc. (également appelé cadre diagnostique)}

\section{Conclusion}

Changements recommandés, nouvelles politiques à adopter pour résoudre le problème (cadre pronostique)

\title{
Garantie
}

\author{
Justifications, appels aux valeurs - \\ raisons pour lesquelles il convient \\ d'intervenir et d'agir sur la situation \\ perturbante (cadre motivationnel)
}

Figure 1 : Cadres d'analyse des problèmes publics (source : Best $2008: 31$, je traduis) 
Une catégorisation des produits discursifs en fonction du modèle de l'analyse des problèmes publics (fig. 1) nous permettra d'évaluer le rôle de la rhétorique de polarisation et de l'insulte dans le mouvement anti-Sarko, et plus particulièrement dans la production de significations et dans la mobilisation d'un consensus. Cette démarche permettra de dégager, dans un premier temps, les éléments constitutifs d'un argument « anti-Sarko » reposant sur la structure donnée-garantie-conclusion. Par la suite, l'analyse des cadres nous permettra d'identifier plus précisément le contenu et l'ampleur de la polarisation mouvement/ennemi, et la manière dont cette polarisation fournit, à travers les insultes, une certaine perception de la cause et de la lutte « antiSarko».

\section{Analyse des cadres}

\subsection{Cadre diagnostique : diabolisation et ridiculisation}

30 Le cadre diagnostique consiste en la représentation symbolique de ce qui est perçu comme "problématique» dans la politique ou la personne de Nicolas Sarkozy. Nous avons trouvé deux catégories principales dans ce cadre, qui empruntent les deux tactiques principales de l'insulte : la diabolisation et la ridiculisation.

\subsubsection{Diabolisation : «Sarko-Facho »}

31 Un premier axe de diabolisation consiste en la représentation de Sarkozy comme un danger. La nature du danger reste relativement vague et variable. L'essentiel est de répéter, de diverses manières, l'idée que Sarkozy constitue une menace. Son nom ou visage sont ainsi intégrés à différentes représentations du mal dans la culture populaire, comme dans l'avertissement sur un paquet de cigarettes, le jeu vidéo (President Evil) ou des personnages connus de films d'horreur tels que Pennywise dans Ça (1990) d'après la nouvelle de Stephen King, ou le vampire Graf Orlok de Nosferatu (1922) :

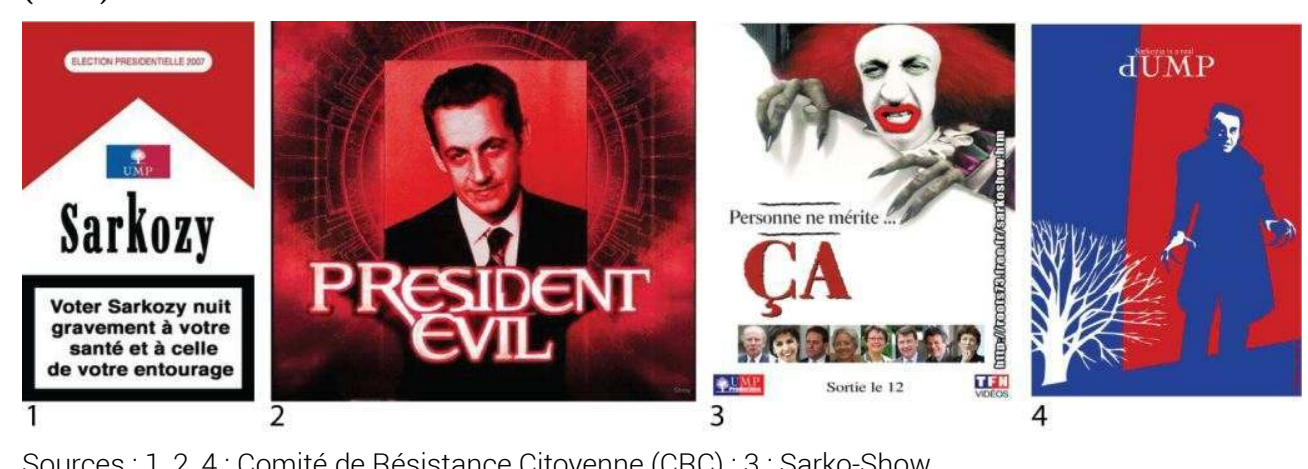

Un deuxième axe de diabolisation est plus précis, et consiste en la représentation de Sarkozy comme un danger pour la démocratie. Ce cadre prend le relais du travail de diabolisation accompli par les adversaires de Sarkozy lors de la campagne présidentielle de 2007 (Amossy \& Koren 2008). Lorsque reprise par la rhétorique du mouvement, la diabolisation se transforme en une représentation caricaturale et concise des idées développées et diffusées peu avant et après les élections. Nous 
pensons, par exemple, à l'appel en faveur de Ségolène Royal qui définit Sarkozy comme :

...la tentation du césarisme contre l'approfondissement de la démocratie, [...] le risque des aventures impériales, du choc des cultures et de l'affrontement des peuples. C'est le candidat de la peur. Des peurs qu'il exploite [...] mais aussi des peurs qu'il inspire (Nouvel Observateur, 1. 3. 2007, je souligne ${ }^{15}$ ).

Loin de disparaître après les élections, cette tendance continue à être développée dans la littérature consacrée au "sarkozysme », notamment dans la théorie du philosophe Alain Badiou qui fait de Sarkozy un "très petit Napoléon ", " petit père du peuple ", «l'homme aux rats », (d'après Freud) et, plus concrètement, associe le "phénomène Sarkozy » au " pétainisme, présentant les abominations subjectives du fascisme (peur, délation, mépris des autres) sans son élan vital » (Badiou 2007 : 20).

Ainsi, dans la rhétorique anti-Sarko ces thèses sont illustrées à travers une série d'affiches comparant ou assimilant Sarkozy à l'une ou l'autre des figures représentatives des régimes non-démocratiques. Le visage ou le corps de Sarkozy sont ainsi collés ou déformés de manière à être associé avec :

Un prototype de dictateur, voire une caricature de dictateur, ou associé à un régime totalitaire :

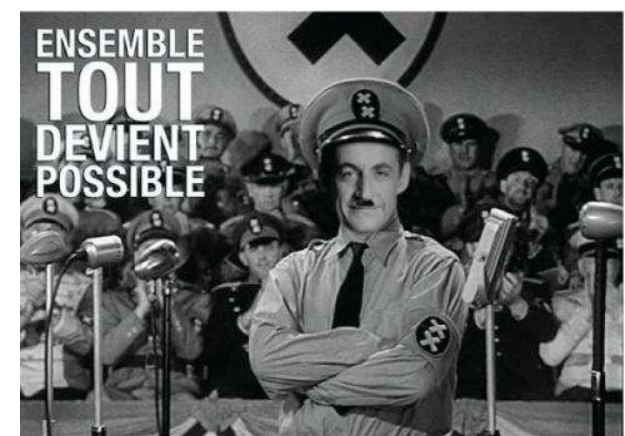

1

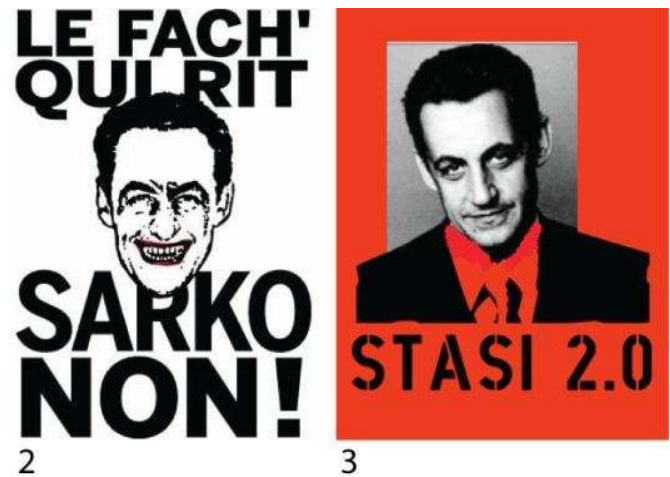

3

Sources : 1 : blog http://antisarko.voila.net/, 2 et 3 : Comité de Résistance Citoyenne (CRC)

Hitler ou le nazisme : 

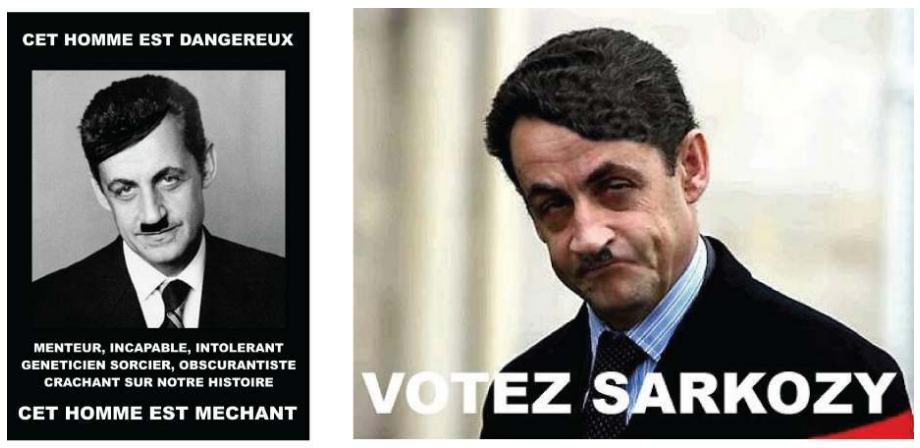

1

2
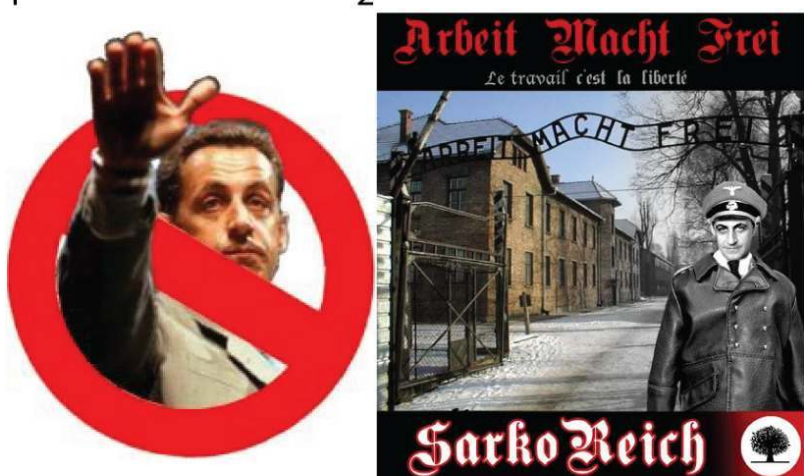

3

4

Sources : 1 : blog http://antisarko.voila.net/, 2, 3, 4 : Comité de Résistance Citoyenne (CRC)

Pétain et le régime Vichy :

Code de l'entrée et du séjour des étrangers et du droit d'asile

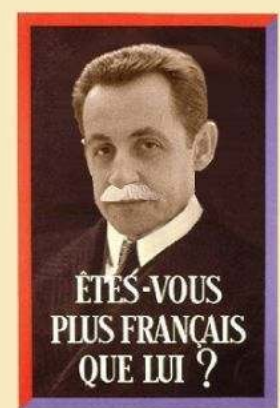

DA VICHY CODE

1

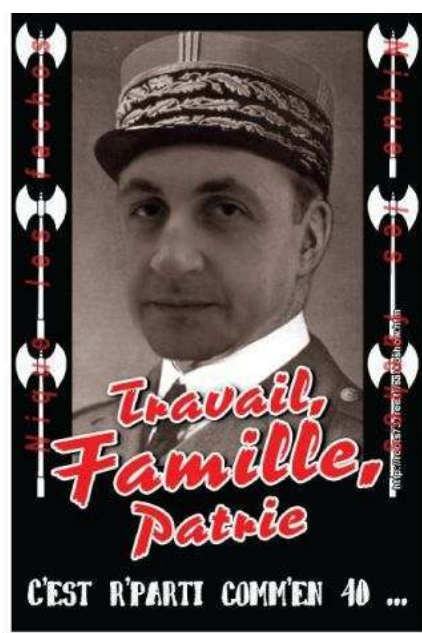

2

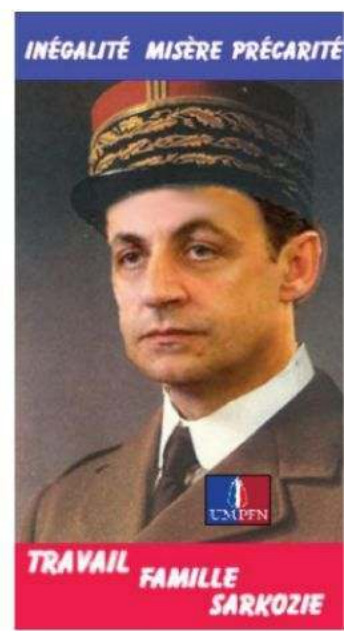

3

Sources : 1 : Le blog de RESO, Réformistes et Solidaires, 2 : Sarko-Show, 3 : blog toutsaufsarkozy.com

Dans ce courant, il existe une sous-catégorie représentant Sarkozy comme une version «légère » de Le Pen ou comme son «fils préféré », comme un Le Pen dissimulé, ou comme une fusion entre les deux, connue sous l'étiquette le « Sarkopen » : 


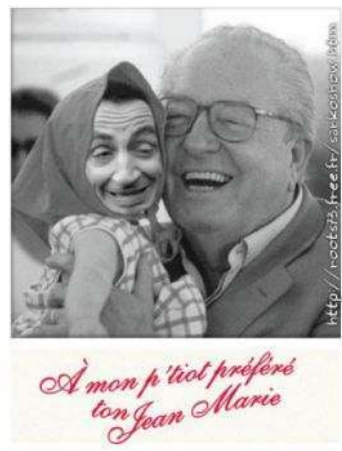

1

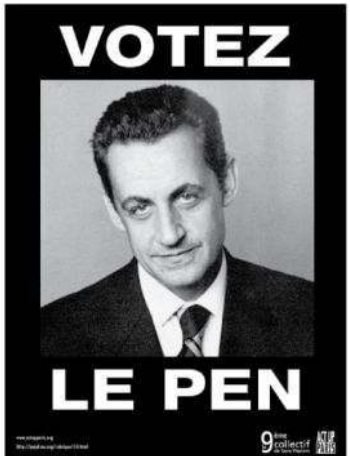

2

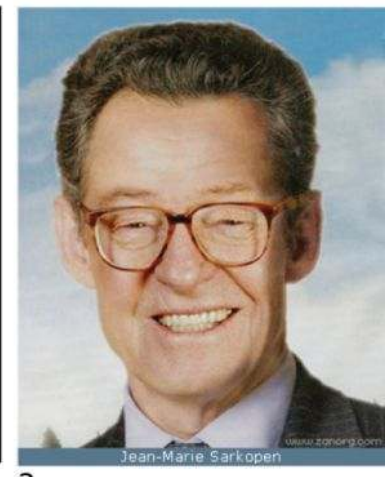

3

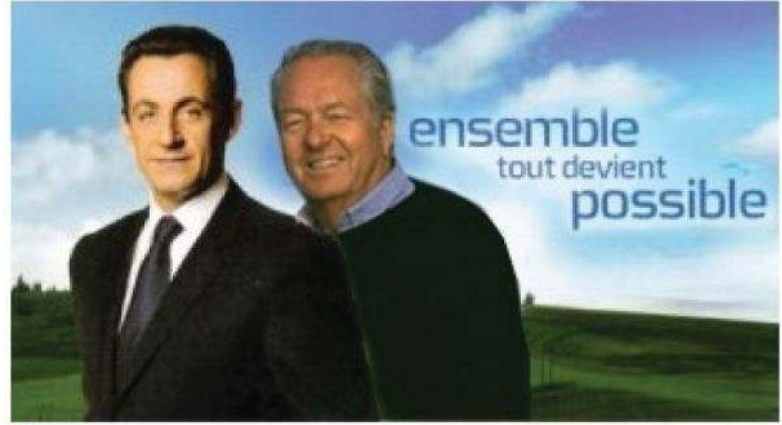

4

Sources : 1 : Sarko-Show, 2, 3, 4 : Comité de Résistance Citoyenne (CRC)

\subsubsection{Ridiculisation : «Pauvre Con»}

Tandis que la diabolisation permet d'attaquer l'aspect idéologique, la ridiculisation cible le personnage de Sarkozy par une représentation particulièrement méprisante voire humiliante de sa personne. Le président est dégradé à travers une déformation de son corps (souvent nu) et de son visage. Les affiches réagissent à différents événements et affaires et leur contenu renvoie souvent à l'actualité courante. Néanmoins, l'effet reste identique: l'apparence de Sarkozy - réelle ou déformée - fait l'objet d'une moquerie acerbe. Nous avons relevé trois catégories principales :

«Petit-Nicolas». Cette catégorie cible les proportions du corps de Sarkozy, en les utilisant pour illustrer son manque de compétence pour le poste de Président : 


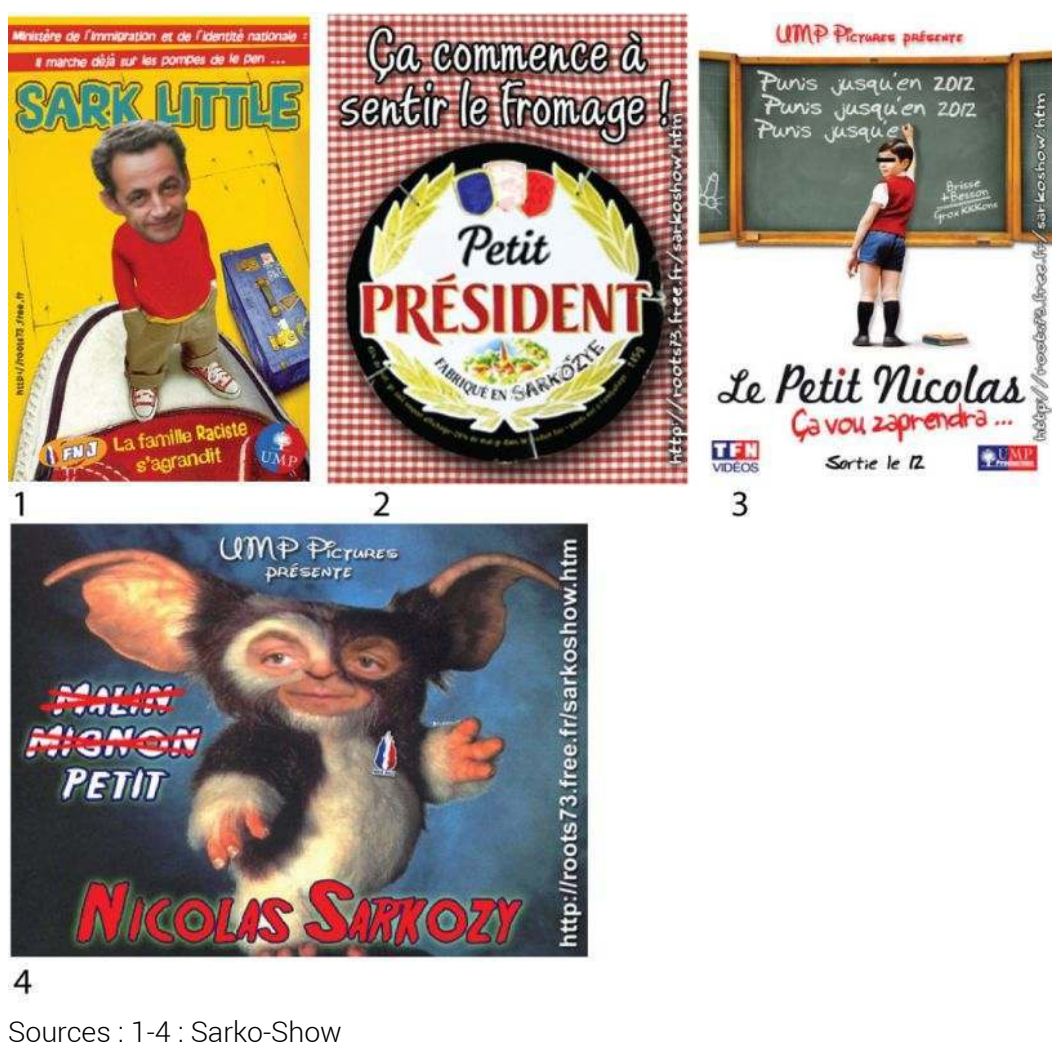

« Sarklown ». Cette catégorie le transforme en clown ou tout autre type de bouffon:
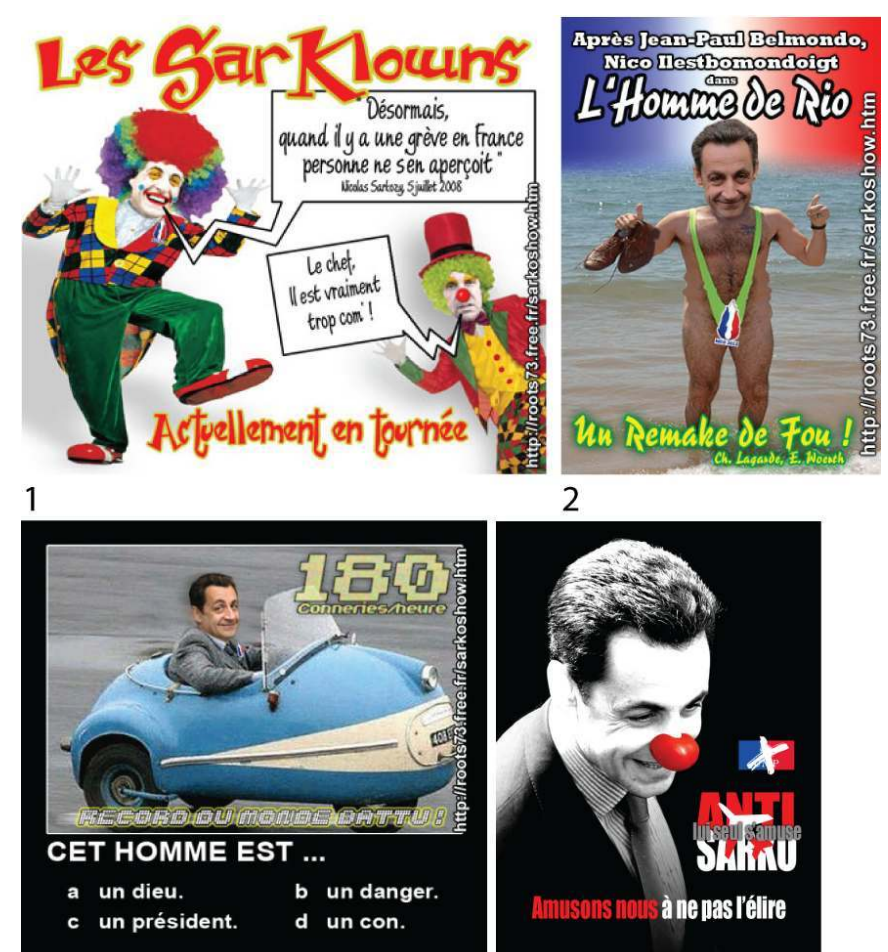

2

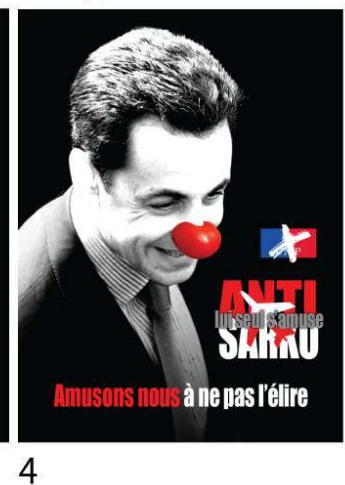

3

Sources : 1-3: Sarko-Show, 4 : antisarko.net

42 Répugnance. Dans cette catégorie Sarkozy est associé à des sentiments de dégoût : son nom ou son image sont liés à des sécrétions corporelles : 


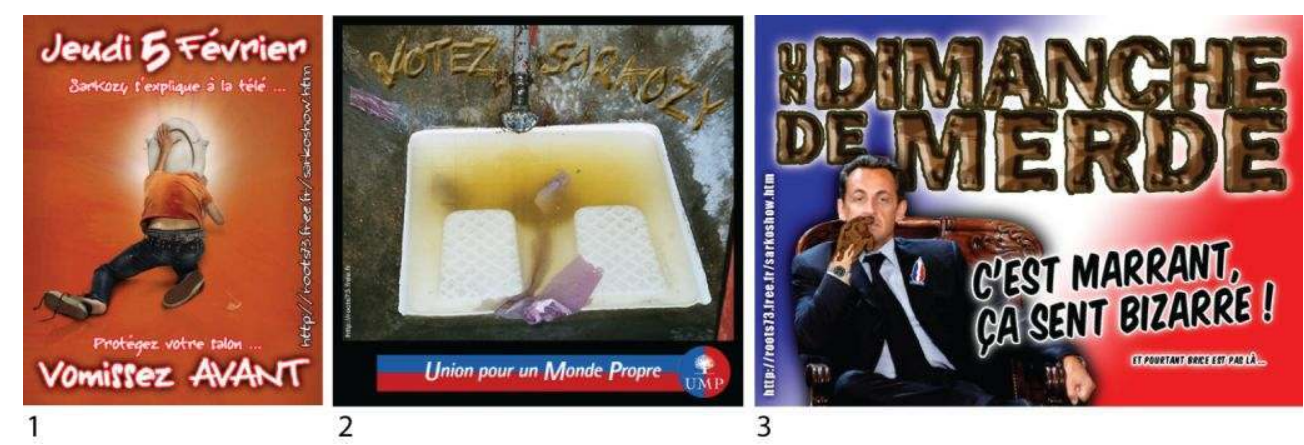

Sources : 1-3: Sarko-Show

43 Un sous-genre de ce type de moquerie consiste à déformer le portrait officiel du Président de la République, illustrant la manière dont le siège officiel du Président est occupé par un « petit » et « con» :

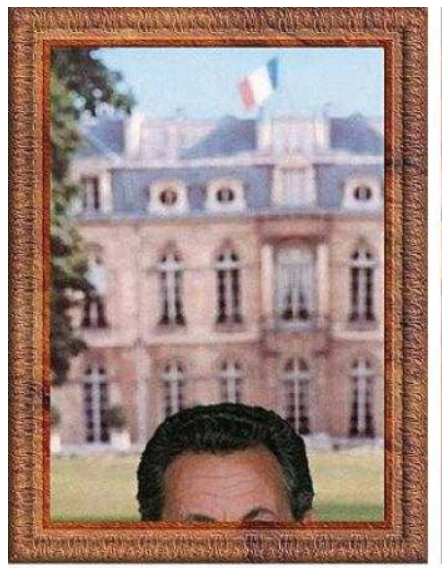

1

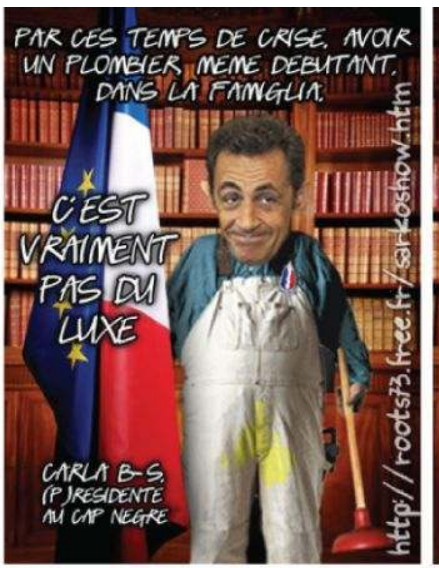

2

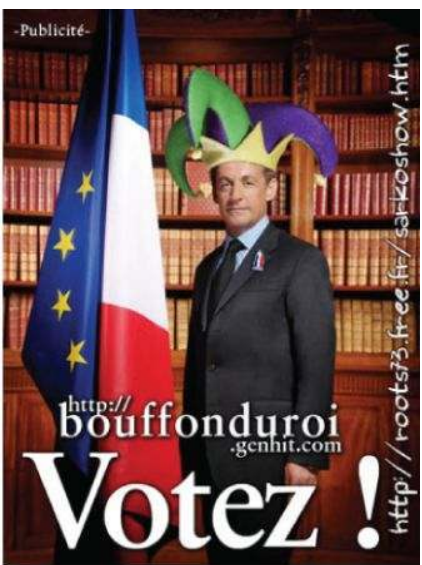

3

Sources : 1 : blog du Sarkomique, 2-3 : Sarko-Show

\section{2. Cadre pronostique : «Casse-toi pauvre con!»}

Le cadre pronostique comprend des représentations symboliques de ce que le mouvement souhaite: la disparition de Sarkozy de la scène publique. Ce cadre est dominé par trois niveaux de représentation. Dans le premier, la fameuse citation de « casse-toi pauvre con! » est détournée et tournée directement contre Sarkozy, qui est ainsi expulsé de l'Elysée : 


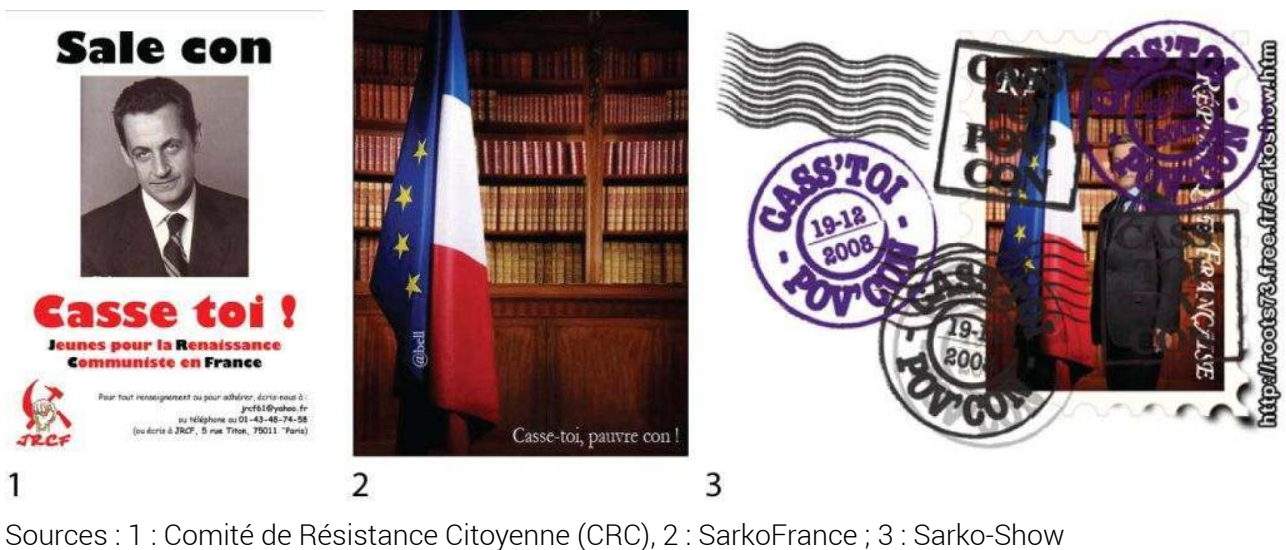

Dans la deuxième catégorie figurent des représentations graphiques de gestes violents ou extrêmement méprisants: Sarkozy devient la cible de coups et autres gestes insolents :

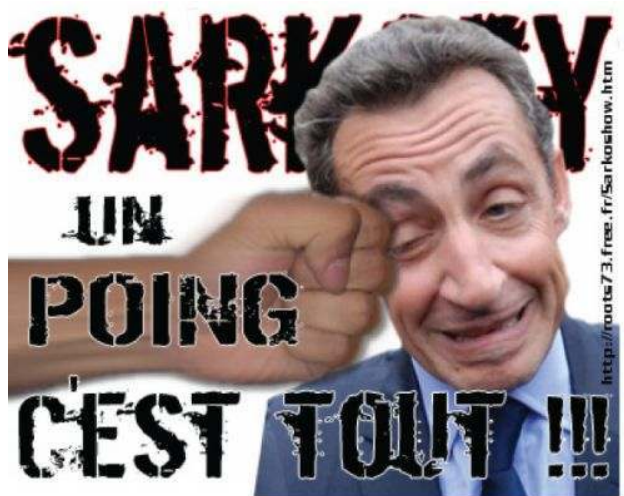

1

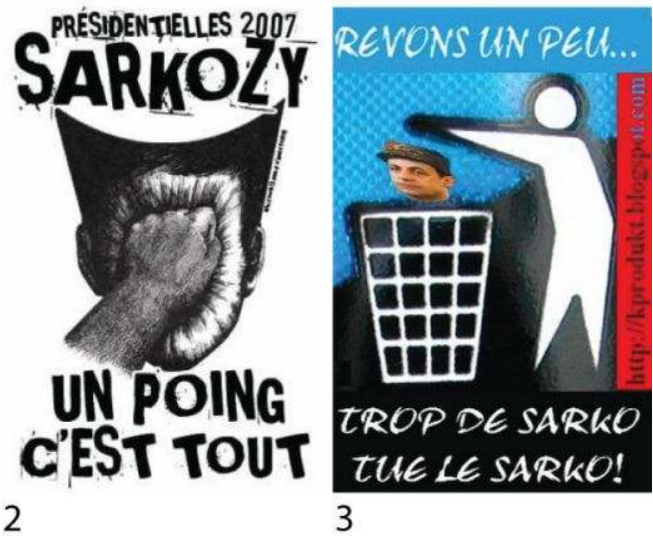

Sources : 1 : Sarko-Show, 2 et 3 : Comité de Résistance Citoyenne (CRC)

La troisième catégorie consiste en une illustration graphique humoristique de la mort de Sarkozy :

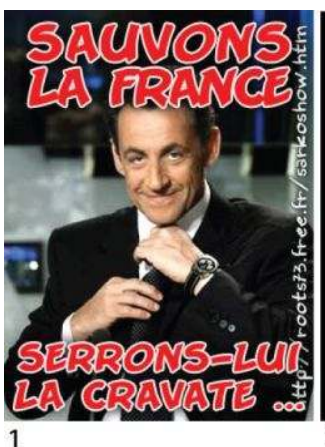

1
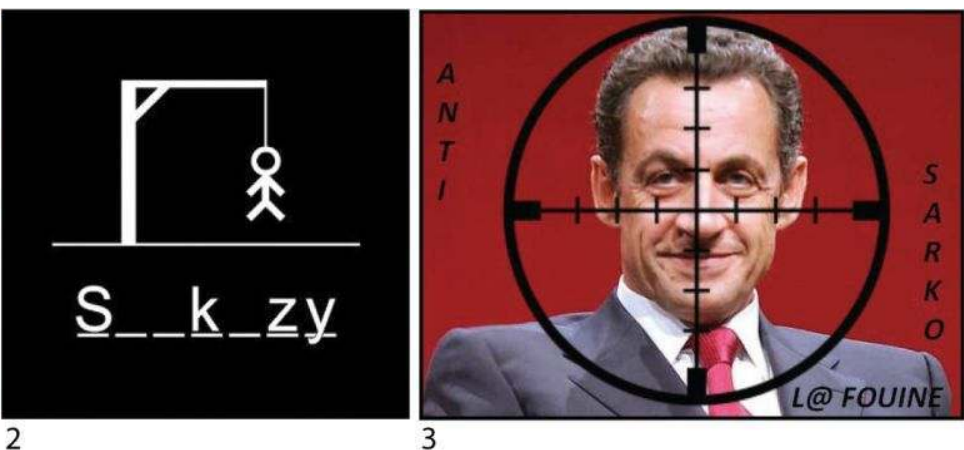

3

Sources : 1 : Sarko-Show, 2 : Comité de Résistance Citoyenne (CRC), 3 : « krakeurs 14 's blog anti sarko » 


\section{3. Cadre motivationnel : «La France d'après »}

47 Le cadre motivationnel permet de justifier la conclusion à travers des appels aux valeurs, sensibilités, croyances et narratifs collectifs. Nous avons trouvé quatre catégories principales.

La première catégorie reprend les slogans de l'UMP lors de la campagne présidentielle 2007, «Imaginons la France d'après » et « Ensemble, tout devient possible ». L'objectif dans le détournement est d'illustrer l'avenir vers lequel se dirige la France sous la présidence de Sarkozy. La France d'après peut être un état policier ou un camp de concentration, elle peut être un paysage démoli, bombardé, elle peut se refléter dans le visage de Sarkozy lui-même, fatigué, malade et vieux ; elle peut devenir un cimetière ou un désert de glace :

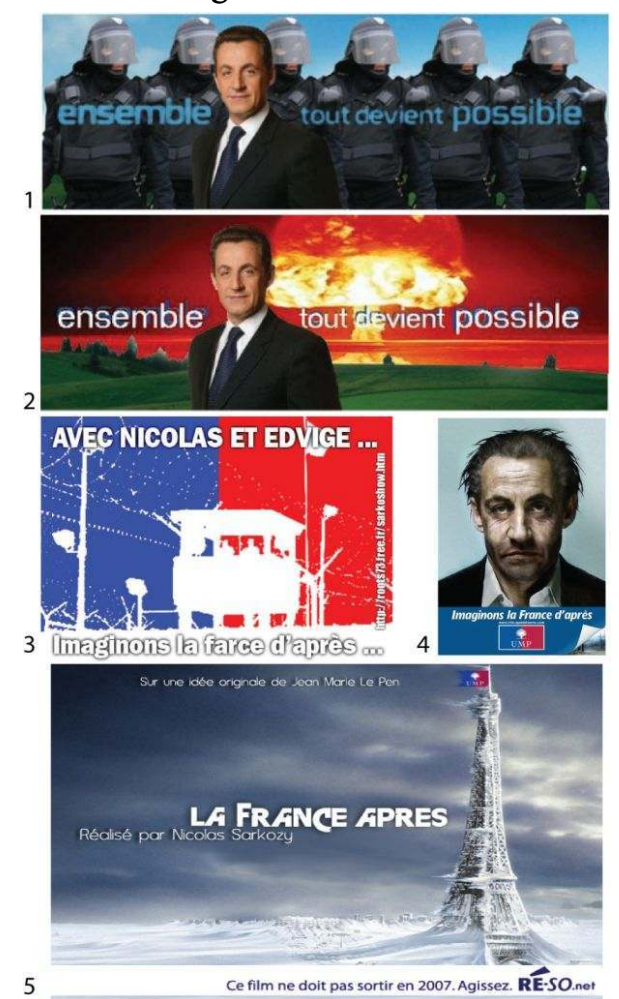

Source : 1-2 : Comité de Résistance Citoyenne, 3 : Sarko-Show, 4 : le blog sarkononmerci.fr/blog, 5 : antisarko.net

49 La deuxième catégorie représente Sarkozy comme un agent des Etats-Unis, une marionnette aux mains des Américains, ou comme un homme politique qui sert plus les intérêts américains que ceux de la France : 


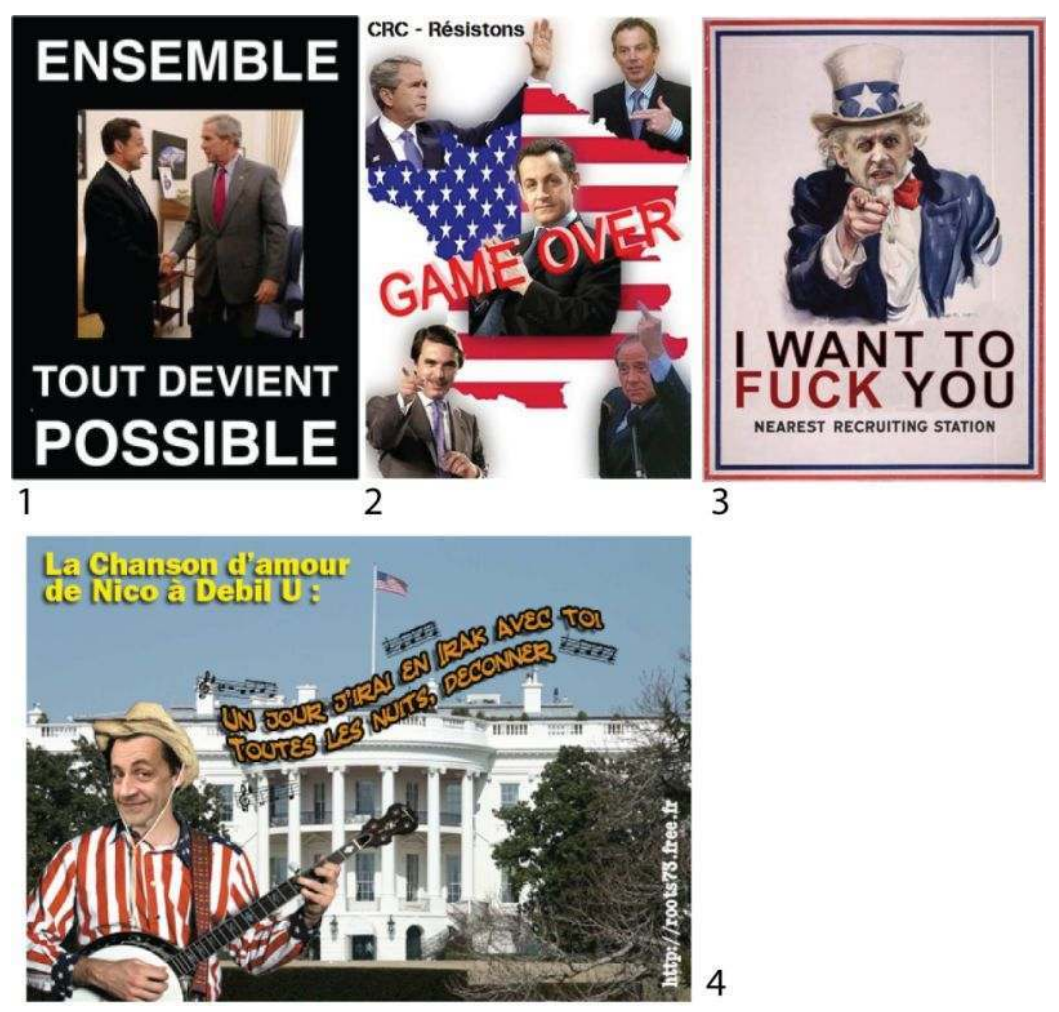

Sources : 1-2 : Comité de Résistance Citoyenne (CRC), 3 : antisarko.net ; 4 : Sarko-Show

La troisième catégorie renvoie aux symboles de la République, détruits ou vidés de leurs sens par Sarkozy. Les symboles peuvent être détournés ou utilisés pour renforcer le sentiment d'urgence :
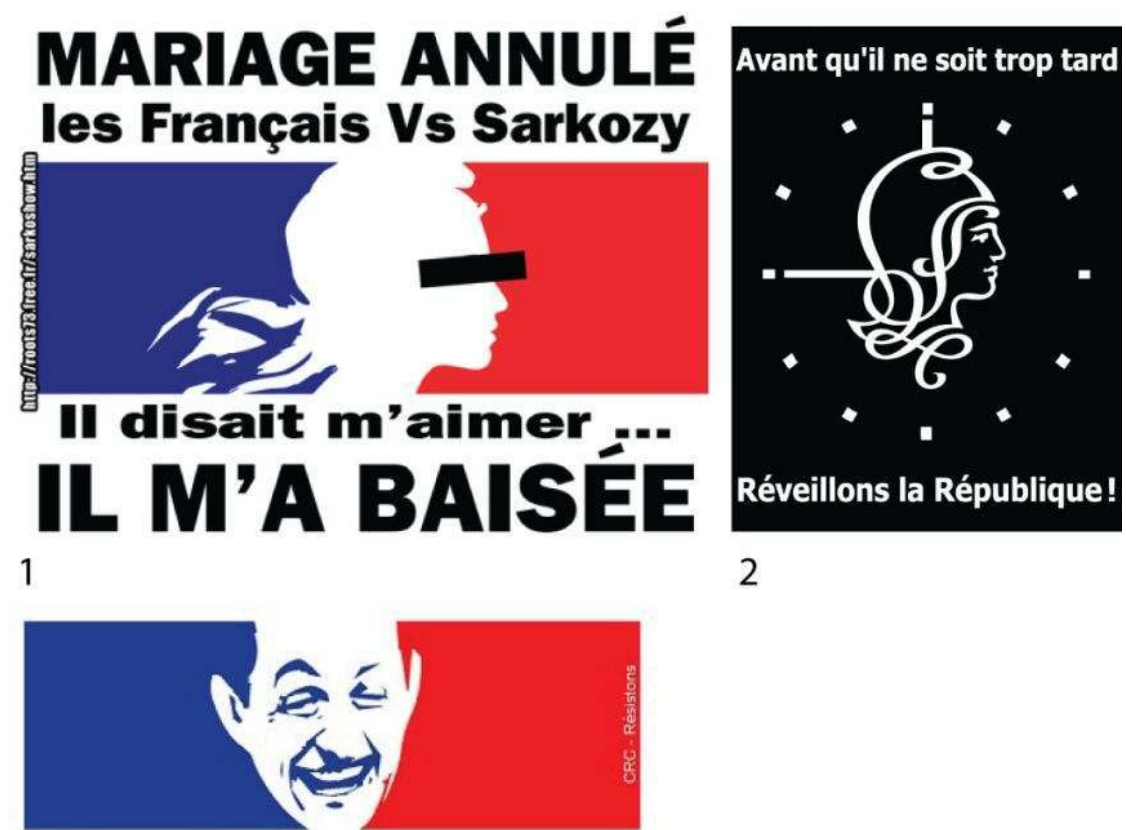

Travail $\cdot$ Famille $\cdot$ Patrie

RÉPUBLIQUE FRANÇAISE 3

Sources : 1 : Sarko-Show, 2 : antisarko.net, 3 : Comité de Résistance Citoyenne (CRC) 
La quatrième catégorie reprend les thèmes et symboles de la Résistance, en attribuant à la lutte contre Sarkozy les valeurs du combat contre le nazisme. Dans cette catégorie sont reproduits et détournés les appels à la Résistance, et la figure tutélaire de De Gaulle est mobilisée :

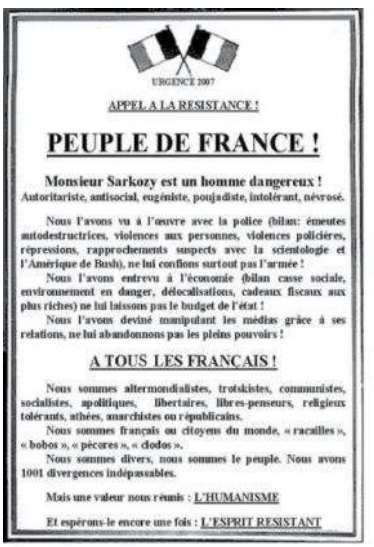

1
APPEL A PARTICIPER A LA RESISTANEE

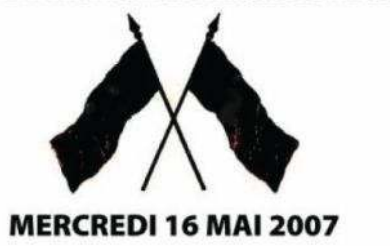

Habillons la France de Noir :

Pour signifier le deuil des libertés. Pour dire que nous ne tolérerons jamais l'eugénisme, l'homophobie, le racisme, etc. Toutes les valeurs antidémocratiques du candidat sarkozy.

"Quand les lois sont injustes, I'obéissance est un crime." Robo Meyrat. 2

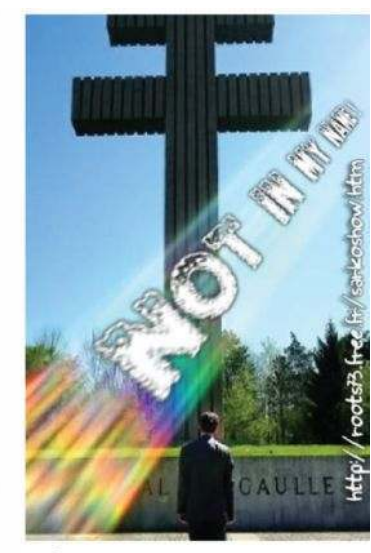

3

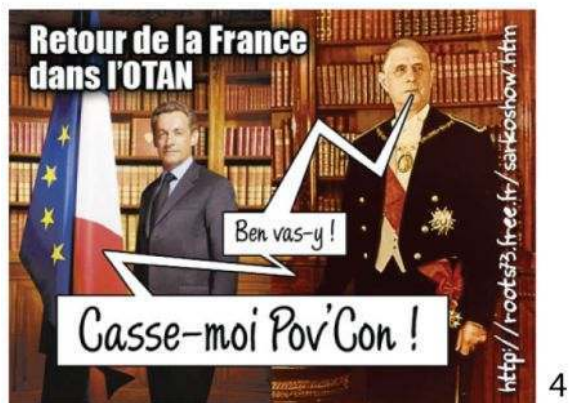

Sources : 1-2 : Comité de Résistance Citoyenne (CRC), 3-4 : Sarko-Show

\section{4. La structure de l'argument « anti-Sarko »}

La catégorisation des cadres des insultes permet tout d'abord de dégager un argument «anti-Sarko » et d'en identifier les éléments constitutifs. Par la diabolisation et la ridiculisation, on apprend que Sarkozy est dangereux, anti-démocratique et incompétent (données) ; Sarkozy doit donc disparaître de la scène publique, il doit être battu, expulsé ou (symboliquement) assassiné (conclusion) ; et ceci dans une optique soucieuse de l'avenir de la France, de son indépendance face à l'américanisme, ainsi que pour défendre les valeurs et les traditions de la République (garantie) : 


$\begin{array}{ll}\begin{array}{l}\text { Données } \\ \text { Sarkozy est dangereux } \\ \text { Sarkozy est anti-démocratique } \\ \begin{array}{l}\text { Sarkozy est incompétent } \\ \text { (cadre diagnostique) }\end{array}\end{array} \\ \\ \qquad \begin{array}{l}\text { Carantie } \\ \text { Sarkozy doit disparaître } \\ \text { (cadre pronostique) }\end{array} \\ & \begin{array}{l}\text { Pour l'avenir de la France } \\ \text { Contre l'américanisme } \\ \text { Pour défendre la tradition et les } \\ \text { valeurs de la République } \\ \text { (cadre motivationnel) }\end{array}\end{array}$

Figure 2 : Structure de l'argument « anti-Sarko »

\section{La figure de « Sarko »}

Nous constatons que tous les cadres sont caractérisés par l'usage de l'humour dans toutes ses variétés : satirique, grotesque, sarcastique et macabre. Cet humour constitue la base du caractère insultant du corpus : au centre de tous les produits discursifs se trouve le rire aux dépens de Sarkozy. Le Président est ainsi systématiquement dépouillé de sa dignité, de son honneur, de son prestige et de son statut public. Les insultes ciblent et minent la stabilité de ces éléments qui constituent une condition sine qua non pour assurer l'autorité politique d'un chef d'Etat dans un régime démocratique.

La diabolisation utilise elle aussi l'humour. Le Sarko-facho qui y est illustré est en même temps menaçant et méprisé. Notons que Sarkozy est présenté comme une version pâle, parodique et ridicule des grands dictateurs de l'histoire : il est « hitlerisé " ou «pétainisé » de manière explicitement artificielle, plus ridicule qu'alarmante. Sa présentation comme bébé de Le Pen ou comme pâle copie de ce dernier, contribue elle aussi à la représentation de Sarkozy comme mauvaise imitation et version pathétique, qui n'arrive même pas à atteindre le niveau de ses prédécesseurs ou de ceux dont il s'inspire.

La ridiculisation de Sarkozy établit une série de traits caractéristiques, visuellement illustrés. Sarkozy est d'abord "petit » : représenté comme un nain, bébé, infantile et immature, l'antonyme caricatural du «Grand Homme »; il est par la suite un " con », une sorte de clown, bouffon, un idiot et imbécile extrêmement risible, toujours plus ridicule qu'alarmant ; et enfin, Sarkozy est un personnage répugnant : il est associé aux sécrétions corporelles de manière à inspirer le dégoût. Le dégoût envers Sarkozy est donc institutionnalisé par les insultes.

Il faut également noter que le corpus est marqué par une forte intertextualité : les catégories ne sont pas entièrement détachées les unes des autres. Nous constatons que, à part le cadre de la ridiculisation, le "petit Sarko " peut figurer dans le cadre de diabolisation (bébé Le Pen) et le "Sarko-Clown» apparaît dans le motivationnel. En fait, c'est à travers le corpus tout entier, cet espace discursif « anti-Sarko » très varié et 
décentralisé, que se dégage un personnage, une sorte de protagoniste qui revient dans tous les produits discursifs. Les insultes créent et reflètent ainsi la figure de "Sarko ", ce « petit con » qui vit dans un « Sarko-Land».

Cette figure de «Sarko» permet de saisir le sens profond des détournements du portrait officiel du Président de la République. La profanation du symbole de l'Etat ne dérive pas de la destruction ou de la déformation de celui-ci, elle est due à la présence de la figure de «Sarko ». Ainsi, l'effet comique est créé par la juxtaposition du symbole officiel et de la figure ridiculisée de Sarkozy. La profanation ne cible pas le symbole luimême et ne représente nullement un rejet des valeurs républicaines comme telles. Au contraire, le détournement du portrait officiel exprime le mépris envers "Sarko », qui contamine l'Elysée. C'est donc "Sarko" qui profane le symbole, et non pas le détournement effectué par le discours contestataire.

C'est également à «Sarko» que s'adresse l'appel "Casse-toi pauv' con!». C'est «Sarko » qui est expulsé de l'Elysée, qui reçoit un coup de poing ou est assassiné. Si elles visent à réaliser l'objectif du mouvement, les illustrations dans le cadre pronostique permettent aussi de manifester un mépris radical envers "Sarko », et de transmettre un sentiment collectif d'irrespect envers l'actuel Président.

\section{Les Français vs. Sarkozy}

Comme nous pouvons le constater à travers le cadre motivationnel, ce sentiment collectif est d'ordre national. Les insultes manifestent le souci de l'avenir de la France, censé être menacé par «Sarko ». Avec lui, la France est en train de devenir malade, de vieillir, de se désintégrer; elle se meurt. L'appel aux sentiments collectifs - voir nationaux - de la France, s'exprime à travers le recours à l'anti-américanisme, ainsi que par l'évocation de l'histoire.

L'association de Sarkozy avec les Etats-Unis et surtout avec la présidence de George W. Bush se nourrit de la protestation contre la guerre en Iraq et de la critique antimondialiste en général. Néanmoins, la représentation de Sarkozy comme celui qui conduit à l'américanisation de la France et qui est fidèle aux intérêts des Etats-Unis, renvoie au sentiment global d'anti-américanisme. Celui-ci s'applique non seulement à Bush (dénommé "Debil-U»), mais aussi aux symboles de la nation américaine (le drapeau, la Maison Blanche) et à la culture américaine (dans nos exemples, la musique country ou la figure de l'« Oncle Sam »). Ainsi, la politique pro-américaine de Sarkozy est transformée par l'insulte en une atteinte aux intérêts de la France, voire en une trahison.

L'évocation de l'histoire de la France, et surtout l'expérience de la Résistance, permet également d'encadrer le mouvement anti-Sarko comme une lutte populaire des Français contre Sarko. Sarkozy est perçu comme celui qui met en cause les valeurs les plus sacrées de la République française, de manière à justifier un « appel aux Français à participer à la Résistance ». La politique de Sarkozy représente une rupture brutale avec la tradition française (comme le retour de la France dans l'OTAN) : on évoque ainsi De Gaulle qui, dans le discours anti-Sarko, se joint au peuple français dans la lutte contre celui qui est censé représenter la tradition gaulliste dans la vie politique.

Ainsi, le recours aux symboles de la République, au sentiment anti-américain, et au patrimoine historique, permet de cibler Sarkozy sans pour autant porter atteinte aux 
institutions républicaines. Les symboles les plus sacrés de la République et de la nation sont déformés et profanés non pas pour rejeter les valeurs qu'ils représentent, mais au contraire pour souligner que, par sa présence sur la scène publique, c'est Sarkozy qui les profane. Loin de diviser «les Français ", cette atteinte aux symboles républicains vise, paradoxalement, à les rassembler autour - et en défense - de ces mêmes symboles.

\section{Conclusions}

L'analyse des cadres nous a permis tout d'abord de dégager, à partir d'un vaste corpus d'insultes, l'argument « anti-Sarko ». Nous avons constaté que le mouvement fait usage de l'insulte pour qualifier Sarkozy de dangereux et d'incompétent, pour exprimer un sentiment collectif de mépris et pour appeler à son expulsion de la scène publique afin de défendre les traditions et les valeurs de la République et de la Nation. Dans cette perspective, l'insulte participe d'une structure argumentative: loin de s'opposer à l'argumentation, comme on le suggère parfois, elle contribue à la construire.

Nous avons également pu voir que les insultes sont à la base d'une rhétorique de polarisation qui établit une division entre l'ennemi et le mouvement. L'ennemi, Sarkozy, est représenté dans le corpus à travers la figure de Sarko, "facho ", " petit ", " con », personnage emblématique méprisable et dégoûtant, qui contamine l'Elysée et profane la présidence et la scène publique par son existence. C'est contre ce "Sarko » que le mouvement se mobilise au nom de tous les Français et s'engage dans une lutte populaire - voire une forme de Résistance - contre celui qui met en cause la France.

Au terme de cette analyse, nous pouvons tirer deux conclusions principales relatives aux fonctions de l'insulte comme outil de cadrage dans la rhétorique interne et externe d'un mouvement social. Dans la mesure où le mouvement « anti-Sarko » est composé de plusieurs regroupements et associations de tendances politiques diverses au sein de la gauche française, l'insulte permet de désigner un ennemi commun qu'il convient d'abattre ensemble. Les attaques répétées contre Sarkozy deviennent la «raison d'être » du mouvement, et participent pleinement à la formation de ses messages. Ce mouvement qui est en train de "se concentrer désormais sur la reconstruction de la gauche ", pose en son centre une cause commune (l'expulsion immédiate de Sarkozy) et une démarche perçue comme une condition préalable à cette reconstruction. L'insulte constitue ainsi la représentation symbolique d'une cause unifiante, et sert d'ancrage sinon de clé de voûte - à tout un mouvement politique. Pour les diverses fractions du mouvement, la figure emblématique de "Sarko" constitue un bouc émissaire, sur lequel sont rejetés tous les maux possibles.

En même temps, et paradoxalement, la stratégie destinée à servir la reconstruction de la gauche accomplit aussi un travail de dépolitisation. Nous constatons, en effet, que l'espace discursif constitué par les insultes, aussi brutales et violentes soient-elles, animent et véhiculent un débat sur les valeurs les plus fondamentales. Il n'est pas impossible d'imaginer que, justement à partir de ce moyen d'expression radical et extrémiste, on puisse parfois discerner un appel à des valeurs plutôt conservatrices, ou à des croyances qui se trouvent à la base de la doxa qui sous-tend l'ensemble de la culture politique française. En faisant appel aux symboles nationaux, les insultes, tout en relevant du langage populaire et de l'obscénité carnavalesque, permettent d'encadrer la lutte comme un mouvement partagé par tous les Français, sans rapport avec leur appartenance politique Ceci permet au mouvement de s'adresser à un vaste 
auditoire et d'élargir sa base de soutien. L'insulte joue ainsi un rôle crucial dans ce qu'on peut définir comme une stratégie rhétorique de dépolitisation de la lutte, en gommant - sur le plan discursif - le clivage politique traditionnel gauche/droite, PS/ UMP, etc. La nature politique du mouvement s'en trouve en effet minimisée, permettant aux adhérents UMP, par exemple, de s'identifier avec la lutte anti-Sarko. Ainsi, ce qui apparaît au premier abord comme un discours polarisant destiné à exclure l'ennemi (et par conséquent à éloigner le camp de ses adhérents) revêt un caractère plutôt inclusif.

\section{BIBLIOGRAPHIE}

Ames, Christopher. 1990. "Power and the Obscene Word: Discourses of Extremity in Thomas Pynchon's ‘Gravity's Rainbow' », Contemporary Literature 31: 2, 191-207

Amossy, Ruth \& Roselyne Koren. 2010. « La 'diabolisation' : un avatar du discours polémique au prisme des Présidentielles de 2007 », Mélanges en l'honneur de Georges Molinié (Paris : Champion)

Badiou, Alain. 2007. De quoi Sarkozy est-il le nom? (Paris : Nouvelles Editions Lignes)

Benford, Robert D. \& David A. Snow. 2000. «Framing Processes and Social Movements: An

Overview and Assessment », Annual Review of Sociology 26, 611-639

Bercoff, André. 2011. La chasse au Sarko (Paris : Editions du Rocher)

Best, Joel. 1990. Threatened Children: Rhetoric and concern about child-victims (Chicago: University of Chicago Press)

Best, Joel. 2008. Social Problems (New-York \& London: Norton)

Bowers, John W., Donovan J. Ochs \& Richard J. Jensen. 1993 [1971]. The Rhetoric of Agitation and Control (Long Gorove, IL: Weaveland Press)

Cefaï, Daniel \& Danny Trom. 2001. « Présentation », Cefaï, Daniel \& Danny Trom (éds). Les formes de l'action collective. Mobilisation dans des arènes publiques (Paris : Editions de l'EHESS), 9-23

Chomsky, Noam. 1969. American Power and the New Mandarins (New-York: Pantheon Books)

Cox, Robert et Christina R. Foust. 2009. « Social Movement Rhetoric », Lunsford, Andrea A., Kirt H. Wilson \& Rosa A. Eberly (éds). The SAGE Handbook of Rhetorical Studies (Los Angeles: Sage Publications), 605-622

Ernotte Philippe \& Laurence Rosier (2004). «L'ontotype : une sous-catégorie pertinente pour classer les insultes? » Langue française 144, 35-48

Fracchiolla, Béatrice \& Claudine Moïse. 2009. «Construction de la violence verbale et circulation des discours : autour du contrat première embauche ", Lopez-Munoz, Juan Manuel, Sophie Marnette, Laurence Rosier \& Diane Vincent (éds.) La circulation des discours, collection Langue et pratiques discursives (Québec : Nota Bene), 103-125 
Jonge, Emmanuel de. 2008. « Pertinence de l'utilisation du modèle de Toulmin dans l'analyse de corpus ", Argumentation et Analyse du Discours 1 [en ligne : http://aad.revues.org/251, consulté le 15 septembre 2011]

Kennedy, Kristen. 1999. « Cynic Rhetoric: The Ethics and Tactics of Resistance », Rhetoric Review $18,26-45$

King, Andrew A. \& Floyd D. Anderson. 1971. « Nixon, Agnew, and the "Silent Majority": A Case Study in the Rhetoric of Polarization », Western Speech 35, 243-255

Klandermans, Bert. 1984. « Mobilization and Participation: Social-Psychological Expansions of Resource Mobilization Theory », American Sociological Review 49 : 5, 583-600

Klandermans, Bert \& Dirk Oegema. 1987. « Potentials, Networks, Motivations, and Barriers: Steps Towards Participation in Social Movements », American Sociological Review 52 : 4, 519-531

Maffesoli, Michel. 2011. Sarkologies. Pourquoi tant de haine(s) ? (Paris : Albin Michel)

Marcuse, Herbert. 1969. An Essay on Liberation (Boston : Beacon Press)

Mucchielli, Laurent. 2011. « La sarko-astro-pseudo-sociologie de Michel Maffesoli », Lectures [En ligne : http://lectures.revues.org/5576, consulté le 15 septembre 2011]

Rosier, Laurence. 2006. Petit traité de l'insulte (Loveral : Editions Labor)

Simons, Herbert W. 1972. «Persuasion in Social Conflicts: A Critique of Prevailing Conceptions and a Framework for Future Research », Speech Monographs 39 : 4, 227-247

Snow, David. 2001. « Analyse de cadres et mouvements sociaux », Cefaï, Daniel \& Danny Trom (éds). Les formes de l'action collective. Mobilisation dans des arènes publiques (Paris : Editions de l'EHESS), 27-49

Snow, David. \& Robert Benford. 1988. «Ideology, Frame Resonance, and Participant Mobilization », International Social Movement Research $1: 1,197-217$

Snow, David \& Robert Benford. 1992. « Master Frames and Cycles of Protest», Morris, Aldon D. \& Carol McClurg Mueller (éds). Frontiers in Social Movement Theory (New Haven, CT: Yale University Press), 133-155

Stewart, Charles J., Craig Allen Smith \& Robert E. Denton, Jr. 2007 [1984]. Persuasion and Social Movements (Long Grove, IL: Waveland Press).

Vanderford, Marsha L. 1989. «Vilification and social movements: A case study of pro-life and pro-choice rhetoric», Quarterly Journal of Speech $75: 2,166-182$

Windt, Theodore Otto Jr. 1972. « The Diatribe: Last Resort for Protest », Quarterly Journal of Speech, $58: 1,1-14$

\section{NOTES}

1. "Pour en finir avec le délit d'outrage ", également connu sous l'appellation « L'appel des 13 », initié par 13 citoyens français condamnés ou poursuivis pour outrage, est diffusé et toujours consultable sur le site de la Ligue des Droits de l'Homme. Sur la question de l'injure en droit, on consultera Lagorgette dans le présent numéro.

2. Cette observation est désormais discutable: dans sa note critique de l'ouvrage de Maffesoli (Sarkologies. Pourquoi tant de haine(s) ?, 2011), Laurent Mucchielli pose que "Aucune donnée historique permettant un minimum de comparaisons, d'où notamment une lancinante discussion 
sur la personnalisation du pouvoir et les attaques ad hominem que subiraient N. Sarkozy de façon exceptionnelle (c'est le titre du livre: "pourquoi tant de haines?»), ce qui est facilement réfutable. Il n'est en effet pas besoin d'une grande culture historique pour savoir que, au contraire, la caricature du Prince est un classique et une constante de la vie politique. Quel responsable politique de premier plan y a échappé dans l'histoire ?» (\$5).

3. Dans le cadre de ce travail nous ne distinguerons pas entre les deux termes du doublet insulte/injure, dont a différenciation par ailleurs problématique ne relève pas de notre questionnement.

4. Cet extrait est tiré de la rubrique « qui sommes-nous? » du site sarkozyland.org (voir : http:// www.sarkozyland.org/index.php?q=node/36, consulté le 15 septembre 2011).

5. Ce crédo apparaît sur la page d'acceuil du site www.antisarko.net (consulté le 15 septembre 2011).

6. Extrait tiré de l'éditorial du premier numéro du Sarkophage, 20 juin 2007 (en ligne : http:// www.pag69.org/article.php3?id_article=614, consulté le 15 septembre 2011).

7. Ibid.

8. Comme, par exemple, les concours d'affiches lancés par le site www.antisarko.net ou la page «ANTI-SARKO - ridiculisons les politiciens !!! » sur Facebook.

9. Voir par exemple http://sarkozix.canalblog.com ou http://sarkostique.over-blog.com.

10. Nous nous référons ici à l'analyse rhétorique des mouvements sociaux telle qu'elle se développe aux Etats-Unis depuis les années 1960. Voir : Cox \& Foust (2009).

11. http://lecrc.canalblog.com/

12. http://roots73.free.fr/sarkoshow.htm

13. http://www.antisarko.net/

14. http://sarkofrance.blogspot.com/, http://www.sarkozyland.org/, http://sarkostique.overblog.com/, http://sarkozix.canalblog.com/.

15. Egalement disponible sur: http://tempsreel.nouvelobs.com/article/20070227.0BS4464/ avant-qu-rsquo-il-ne-soit-trop-tard.html, consulté le 15 septembre 2011.

\section{RÉSUMÉS}

Sur la base d'un vaste corpus relatif au mouvement « anti-Sarko » en France, cet article explore les significations et les fonctions de l'insulte dans la rhétorique de protestation. Exploitant les méthodes de l'analyse des cadres, l'article classifie d'abord les différentes catégories d'insultes qui caractérisent le genre humoristique " anti-Sarko », puis démontre comment ces catégories construisent ensemble l'argument « anti-Sarko » de base. L'article montre que les insultes jouent un rôle central dans le cadrage de la cause et des motifs du mouvement. La rhétorique des insultes permet à ce mouvement relativement fragmenté et divisé de se rassembler autour d'un ennemi commun, et de projeter une série de valeurs partagées avec lesquelles le «français moyen " peut facilement s'identifier.

Drawing on a large corpus from the French "Anti-Sarko" movement, this article explores the meanings and functions of the insults in the rhetoric of protest. Using Frame Analysis methods, the article first classifies different categories of insults in the "anti-Sarko" humoristic genre, and then demonstrates how these categories form together the fundamental "anti-Sarko" argument. The article argues that the insults play a central role in the movement's effort to frame its cause 
and motives. The rhetoric of insults also enables the relatively fragmented and divided movement to reunite against a common enemy, and to reflect a shared set of values with which the "average-French" could easily identify.

INDEX

Mots-clés : cadrage, humour, insulte, rhétorique des mouvements sociaux, Sarkozy (Nicolas), violence verbale

Keywords : framing, humor, insult, Sarkozy (Nicolas), social movement rhetoric, verbal aggression

\section{AUTEUR}

EITHAN ORKIBI

Université de Tel-Aviv et Université Bar-Ilan, ADARR 